\title{
Evidence for similar early but not late representation of possible and impossible objects
}

\author{
Erez Freud $^{1,2}$ *, Bat-Sheva Hadad ${ }^{3}$, Galia Avidan ${ }^{1,2}$ and Tzvi Ganel ${ }^{1,2}$ \\ ' Department of Psychology, Ben-Gurion University of the Negev, Beer-Sheva, Israel \\ ${ }^{2}$ Zlotowski Center for Neuroscience, Ben-Gurion University of the Negev, Beer-Sheva, Israel \\ ${ }^{3}$ Edmond J. Safra Brain Research Center, Faculty of Education, University of Haifa, Haifa, Israel
}

Edited by:

Gregory Zelinsky, Stony Brook

University, USA

\section{Reviewed by:}

Corrado Caudek, Università di

Firenze, Italy

Robert G. Alexander, Stony Brook

University, USA

${ }^{*}$ Correspondence:

Erez Freud, Department of

Psychology, Ben-Gurion University

of the Negev, The Abraham Ben

David Ohayon, Behavioral Sciences

Complex, P.O.B. 653, Beer-Sheva,

Israel

e-mail: erezfreud@gmail.com
The perceptual processes that mediate the ability to efficiently represent object 3D structure are still not fully understood. The current study was aimed to shed light on these processes by utilizing spatially possible and impossible objects that could not be created in real 3D space. Despite being perceived as exceptionally unusual, impossible objects still possess fundamental Gestalt attributes and valid local depth cues that may support their initial successful representation. Based on this notion and on recent findings from our lab, we hypothesized that the initial representation of impossible objects would involve common mechanisms to those mediating typical object perception while the perceived differences between possible and impossible objects would emerge later along the processing hierarchy. In Experiment 1, participants preformed same/different classifications of two markers superimposed on a display containing two objects (possible or impossible). Faster reaction times were observed for displays in which the markers were superimposed on the same object ("object-based benefit"). Importantly, this benefit was similar for possible and impossible objects, suggesting that the representations of the two object categories rely on similar perceptual organization processes. Yet, responses for impossible objects were slower compared to possible objects. Experiment 2 was designed to examine the origin of this effect. Participants classified the location of two markers while exposure duration was manipulated. A similar pattern of performance was found for possible and impossible objects for the short exposure duration, with differences in accuracy between these two types of objects emerging only for longer exposure durations. Overall, these findings provide evidence that the representation of object structure relies on a multi-level process and that object impossibility selectively impairs the rendering of fine-detailed description of object structure.

Keywords: impossible objects, coarse-to-fine, object-based attention, 3D structure, object recognition

\section{INTRODUCTION}

Throughout our daily lives we recognize thousands of objects quickly and accurately. One particular requirement for successful object recognition is the extraction of object 3D structure, which enables humans to recognize objects despite enormous changes across viewpoint and size (Marr, 1982). To date, the exact nature of the cognitive and neural mechanisms that support the representation of object 3D structure is still not fully understood.

In the present study we utilized a special type of visual illusion known as "impossible objects" to further unravel the perceptual processes that mediate the representation of object 3D structure. Impossible objects are defined as $2 \mathrm{D}$ drawings that represent 3D objects that could not exist in real 3D space (Penrose and Penrose, 1958; Figure 1). At the 2D level, the physical differences between possible and impossible objects are minor; however, the 3D interpretation of the structure of these two object categories is markedly different. Hence impossible objects offer a unique opportunity to test the representation of object 3D structure while other visual properties are well controlled.

Impossible objects reflect internal inconsistencies between the global and local information embedded in the stimulus. Specifically, while the local elements that compose the object are completely valid, the perceived global 3D structure is incoherent. Such inconsistencies were previously investigated in the context of different depth cues such as structure from motion, disparity and perspective lines (e.g., Domini et al., 1998, 2006; Di Luca et al., 2004; Knill and Pouget, 2004; Burge et al., 2005; Knill, 2007). For example, Di Luca et al. (2004) found that the perception of local surfaces that were defined by 3D structure from motion, were biased by the global properties of the stimuli. These findings strongly suggest that the perceived 3D shape is generated based on the integration of various cues, and that the effect of global information can be considered as automatic in nature. Accordingly, previous studies that investigated the perceptual processes that mediate the representation of object impossibility have found 


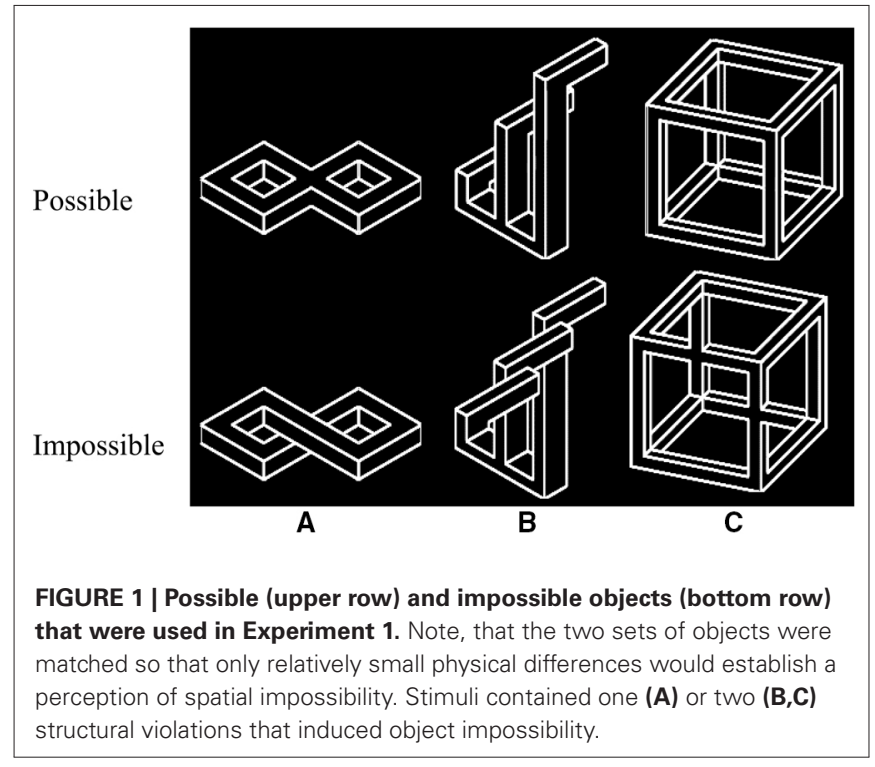

that the visual system is highly susceptible to detect object impossibility even when it is task irrelevant. In particular, it was found that a set of cortical regions along the dorsal and ventral visual streams is highly sensitive to object impossibility (Freud et al., 2015). Additionally, a behavioral study showed that 4 month old infants looked longer at impossible objects compared to possible objects (Shuwairi et al., 2007). Similar sensitivity was found even in non-human animals (i.e., newly hatched chicks) that exhibited a spontaneous preference to possible objects (Regolin et al., 2011).

On the other hand, other studies also showed similarities in the perceptual and neural mechanisms that mediate the representations of possible and impossible objects. Specifically, similar Garner interference effects (i.e., worse performance in a classification task of a given object's dimension when an irrelevant dimension is varied; Garner and Felfoldy, 1970) was found for possible and impossible objects, suggesting that the two object categories were processed in a holistic fashion (Freud et al., 2013a). Additionally, in fMRI studies, similar adaptation patterns were found for possible and impossible objects (Habeck et al., 2006; Freud et al., 2013b).

These seemingly contradicting findings imply that under some conditions, the visual system is insensitive to object impossibility, however, under other conditions, it may still retain great susceptibility to object $3 \mathrm{D}$ structure and to the inconsistencies between the global and local information. Importantly, as noted above, impossible objects, just as possible ones, possess valid depth cues which are based on fundamental Gestalt shape attributes (e.g., object closure, distinguishable surfaces, and volume properties) (Koffka, 1935; Kovacs and Julesz, 1993; Geisler et al., 2001). Thus, it is reasonable to assume that initial representations of these objects could be successfully generated. Consequently, the spatial ambiguity embedded in impossible objects may impair higher perceptual processes.

We recently tested this view in the context of a behavioral studies focused on the role of spatial frequency in the representation of possible and impossible objects. These studies already alluded to a dissociable role of different spatial frequency ranges in object recognition. Specifically, it has been demonstrated that low spatial frequency (LSF) information conveys global properties of the objects and supports the generation of their initial coarse representation, while high spatial frequency (HSF) information is processed later in time and supports the rendering of fine-grained description of these objects (e.g., Sugase et al., 1999; Bar, 2003; Bar et al., 2006). Consistently, using the Garner speeded classification task (Garner and Felfoldy, 1970) we showed that a similar processing style (i.e., holistic representation) was applied to both possible and impossible objects following the filtering of their HSF content. Contrary, when LSF information, which mainly supports the initial representation, was filtered out, the responses to the two object categories were dissociated. Particularly, possible objects were still processed holistically, while impossible objects were processed in an analytic-local fashion and were no longer perceived as unified objects (Freud et al., 2014).

The current study was aimed to further investigate the perceptual processes that mediate the representation of object $3 \mathrm{D}$ structure. In two experiments, we tested two basic predictions stemming from our working hypothesis. Experiment 1 tested whether impossible objects are organized and perceived as unified wholes similarly to possible objects using an established perceptual measure of object-based processing style. Experiment 2 further examined whether the observed differences between object categories rely on early or on late perceptual processes.

\section{EXPERIMENT 1}

The purpose of Experiment 1 was to test whether possible and impossible objects share similar initial perceptual processes using an established behavioral paradigm. Specifically, we applied the classical "object-based attention" paradigm originally devised by Duncan (1984). In this paradigm participants are asked to perform simple perceptual classifications of two visual elements in the display (e.g., same/different classifications) and the typical finding is of better performance when the two to be judged features, belong to the same object rather than placed on two different objects. The common interpretation of this result is that visual attention operates on object-based representations, and is therefore constrained by perceptual organization processes of object segregation (Duncan, 1984; Kimchi et al., 2007).

The main hypothesis was that impossible objects would have a similar object-based processing style as possible ones. Specifically, we hypothesized that if the visual system constructs a valid initial representation for impossible objects, similarly to possible objects, these perceptually organized representations should capture attention so that targets placed on the same object would be more efficiently processed than when placed on two different impossible objects.

\section{MATERIALS AND METHODS \\ Participants}

Thirty students with normal or corrected to normal vision participated in the experiment. They all provided informed consent to participate in the experiment and received $\$ 5$ equivalent for their participation. The data of one participant was discarded from the analysis due to exceptionally low accuracy (less than 
$70 \%$ compared to average of $93.6 \%$ of the other participants). All experimental procedures were approved by the ethics committee of the Psychology Department at Ben-Gurion University of the Negev.

\section{Apparatus and stimuli}

Three pairs of possible and impossible objects were used as experimental stimuli (Figure 1). Possible and matched impossible objects were identical except for one (Figure 1A) or two features (Figures 1B,C) inserted to make the object's global 3D structure impossible. All pairs were used in previous experiments (Freud et al., 2013b, 2015).

In each trial, a pair of objects (possible or impossible) was presented (Figure 2). Stimuli subtended a visual angle of approximately $12^{\circ}$ and were presented on a 19 inch computer screen $(1024 \times 768$ resolution; refresh rate of $60 \mathrm{HZ})$. Additionally, two red circles-filled or empty (a visual angle- $0.3^{\circ}$ each) were superimposed on different locations on the objects depending on the specific experimental condition (same object/different objects). The circles were located on clear surfaces while avoiding more ambiguous locations such as object's counters. Critically, the distance between the two circles was kept constant across trials regardless of their exact placement on the screen.

\section{Design}

Object possibility (possible/impossible), targets location (on different objects/on the same object), object orientation (horizontal/ vertical; normal/mirror), and correct decision (same/different) served as within-subjects variables with each combination occurring on an equal number of trials (see Figure 2). The results were collapsed across object orientation and correct decision after preliminary analyses that ensured that these variables did not affect

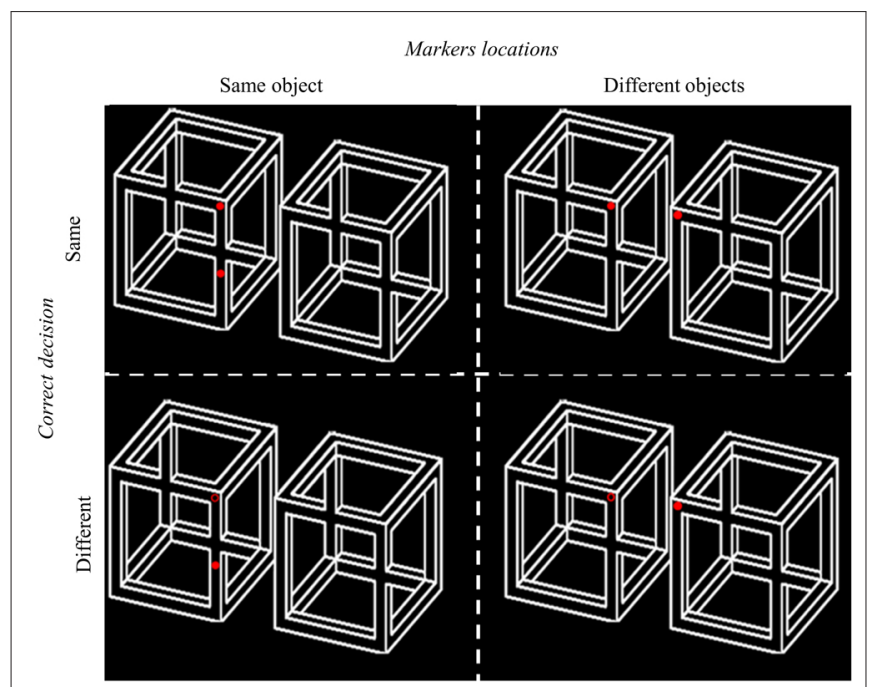

FIGURE 2 | Examples of stimuli presented in Experiment 1. On each trial, two red circles were superimposed on the display and participants were asked to judge if the circles are same or different (rows). The locations of the circles varied across trials such that for half of the trials the circles were located on the same object (left column) and for the other half the circles were located on different objects (right column). Display orientation was counterbalanced across trials (see text for details). the interaction between possibility and target location $\left[F_{s}<1\right]$. Additionally, object exemplar served as a between-subject variable (three levels; Figure 1). Both accuracy and reaction times were recorded.

\section{Experimental procedure}

Participants were instructed to report as accurately and as quickly as possible whether the two red circles in each trial were identical (filled or empty) or different (one filled and one empty; Figure 2) regardless of their locations. Object type (possible/impossible) was not explicitly mentioned to avoid possible attentional confounds.

Following four practice trials in which feedback was provided that were discarded from further analysis, participants were presented with 256 experimental trials distributed equally and randomly between the different conditions. The trials were self-generated; each trial began with a fixation point $(300 \mathrm{~ms})$, followed by the experimental display $(1000 \mathrm{~ms})$ and a blank screen (1500 ms). Responses were recorded using a keyboard in which two stickers were attached to the designated response keys.

\section{RESULTS}

Accuracy rate was high (93.6\%). A repeated measures analysis of variance (ANOVA) revealed no accuracy-based effects of object type or of target locations [all $F$ s $<1$ ]. Analysis was therefore focused on RTs for correct trials.

Latencies shorter than $200 \mathrm{~ms}$ and longer than $1000 \mathrm{~ms}$ were considered outliers and were removed from further analysis $(4 \%$ of the correct responses). The reaction time data is presented in Figure 3. As can be seen, when targets were located on the same object, reaction times were faster compared to trials in which the targets were located on two different objects. A repeated measure ANOVA revealed a main effect of target location $\left[F_{(1,26)}=5.93\right.$, $\left.p<0.05, \eta_{\mathrm{p}}^{2}=0.18\right]$ with no interactions with object possibility $\left[F_{(2,26)}<1\right]$, or specific exemplar $\left[F_{(2,26)}=1.5, p>0.2\right]$. The three-way interaction was not significant $\left[F_{(2,26)}=1.6, p>0.2\right]$. Importantly, planned comparisons confirmed that the benefit of

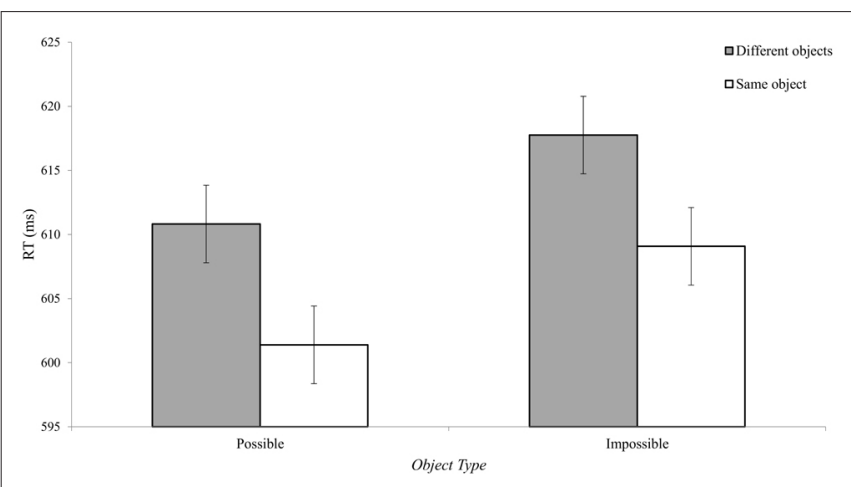

FIGURE 3 | Reaction times in Experiment 1. Participants responded faster when the circles were located on the same object (an object-based attentional effect) regardless of object possibility. In addition, shorter reaction times were found for possible compared to impossible objects. Error bars represent confidence intervals as calculated for repeated measure ANOVAs for the effect of circles locations (Jarmasz and Hollands, 2009). 
"same object" was evident for possible $\left[F_{(1,26)}=5.42, p<0.05\right]$ and marginally for impossible objects $\left[F_{(1,26)}=3.85, p=0.06\right]$.

Interestingly, despite the fact the impossible objects drew attention in a similar fashion to possible objects, a main effect was found for possibility, with slower reaction times for impossible compared to possible objects $\left[F_{(1,26)}=12.8, p<0.001\right.$, $\left.\eta_{\mathrm{p}}^{2}=0.33\right]$. A marginal interaction was also found between exemplar and object possibility $\left[F_{(2,26)}=2.7, p=0.08\right]$, indicating a more robust effect for some exemplars compared to others. Nevertheless, a similar trend of slower reaction times for impossible objects was found for all exemplars.

Finally, for each participants only one exemplar was used along the experiment to increase statistical power. A similar procedure was also used in previous studies that examined the sensitivity of the visual system to object impossibility (e.g., Shuwairi et al., 2007; Freud et al., 2014). However, repeated presentations of these objects might reduce their perceived impossibility. To examine this issue, we have reanalyzed that data separately for the first and the second part of the experiment. This analysis revealed that in addition to the main effect of object possibility $\left[F_{(1,26)}=14.6, p<0.001\right]$, a main effect for experiment's part was found $\left[F_{(1,26)}=29.6, p<0.001\right]$, such that faster RT were found in the second part of the experiment compared to the first half. Importantly however, these two factors did not interact with one another $[F<1]$, suggesting that despite the repeated presentations of the same exemplar, the visual system did not treat the impossible object as different (i.e., more possible) at the second part of the experiment.

\section{DISCUSSION}

The results of Experiment 1 show that impossible objects draw visual attention in a similar fashion to possible objects. This finding suggests that despite their unusual spatial structure, impossible objects share similar perceptual organization processes to those mediating the representation of possible objects. This sameobject benefit is observed despite the relative complexity of the stimuli compared to previous studies (e.g., Moore et al., 1998; Zemel et al., 2002; Drummond and Shomstein, 2010). Moreover, for both object categories, the attentional capture occurred automatically with an object-based effect obtained despite the fact that the task was irrelevant to object structure (Kimchi et al., 2007).

One can argue that Gestalt cues such as common region, closure, etc., which are evident for both object categories, could account for the object-based effects observed for possible and impossible objects. We cannot fully disqualify this alternative. Still, a recent study indicates that a unified internal representation of an object, rather than a mere collection of its features, is required to support the object-based facilitation effect (Naber et al., 2011). Thus, the object-based effect found in Experiment 1 for impossible objects implies that object representations exists for impossible objects despite the spatial incoherency inherent to this object category.

Note that the two circles were located on the same 2D structurally possible surface. This was done to ensure that the perceptual requirements were equal between the possible and impossible conditions and that the circles were located on visually identical locations for the two object categories. Presentations of the circles on different surfaces for possible and impossible objects could have therefore resulted in an unwarranted confound which is avoided by the present design. However, this issue may raise an alternative explanation according to which the attentional selection was mediated by the processing of the $2 \mathrm{D}$ plane of the objects rather than of $3 \mathrm{D}$ information. Nevertheless, several lines of evidence are not in accord with such an alternative explanation. First, in the present study a main effect was found for object possibility, indicating that participants still processed object 3D information despite being irrelevant to the task in hand. Additionally, previous works from our lab and from other labs have shown that object 3D structure is processed in an automatic fashion (Goldfarb and Tzelgov, 2005; Freud et al., 2013a) and that object possibility is processed even when object category is irrelevant (Shuwairi et al., 2007; Regolin et al., 2011; Freud et al., 2014, 2015). Hence, it is unlikely that the participants independently processed the $2 \mathrm{D}$ plane in a unified manner, while other portions of the stimulus were not integrated.

In addition to the main result of similar object-based attention effects for the two object types, we also found some differences between the processing of the two object categories. In particular, slower classification times were found for impossible compared to possible objects. The nature of the underlying perceptual mechanisms that mediate the delayed responses for impossible objects was further investigated in Experiment 2.

\section{EXPERIMENT 2}

The overall slower reaction times found for impossible objects in Experiment 1 could be accounted for by two fundamentally different plausible perceptual mechanisms. According to a bottomup scheme, object structural impossibility can be encoded by a simple-to-complex architecture, based on hierarchical shape detectors. According to such an alternative view, the spatial ambiguity embedded in impossible objects impairs the initial perceptual organization of this object category. Only later in time along the hierarchy of visual processing, local valid depth cues are used to support object-based representation. Note, that previous research has shown that such models could account for the representation of complex stimuli such as faces and objects, and could also account for effect of global-configural organization (Jiang et al., 2006).

On the other hand, according to our working hypothesis, the initial representation of impossible objects is intact, and only later in time along the hierarchy of visual processing object impossibility is represented. Experiment 2 utilized a different experimental design aimed to distinguish between these two alternatives. To this end, we tested when along the processing hierarchy the perceived differences between the object categories start to emerge by manipulating exposure duration. We used two exposure durations. In the short exposure duration the stimulus was presented for $85 \mathrm{~ms}$ as previous studies suggested that this duration is the minimal time required for successful object recognition (GrillSpector and Kanwisher, 2005; Sayres and Grill-Spector, 2006). The long exposure duration was set to $986 \mathrm{~ms}$ to allow full processing of object structure.

Importantly, in order to test object processing per se, we adopted a new task in which only a single object was presented in 
each trial. Participants were required to judge whether two circles are located on the object or whether one of the circles is located outside the boundaries of that object (Figures 4A,B).

According to our working hypothesis, the initial perceptual processes subserving the processing of possible and impossible objects should be similar and therefore no differences were expected to be found between the two object categories for short exposure durations. On the other hand, for the longer exposure duration, we predicted that there would be enough time for the incoherency of the structural information embedded in impossible objects to be processed, which should in turn impede the fine-grained representation of this object category.

\section{MATERIALS AND METHODS Participants}

Twenty six students (none of whom participated in Experiment 1) with normal or corrected to normal vision participated in the experiment. They all provided informed consent to participate in the experiment and received $5 \$$ equivalent for their participation. The data of two participants was discarded from the analysis due to exceptionally low performance in the short exposure time condition $(<50 \%)$.

\section{Apparatus and stimuli}

Seven pairs of possible and impossible objects were used in Experiment 2. Each trial display contained only one object that subtended a visual angle of approximately $9^{\circ}$ visual angle, and was presented on a 19 inch monitor (resolution $1024 \times 768$; refresh rate of $60 \mathrm{HZ}$ ). Two red circles (visual angle: $0.3^{\circ}$ ) were superimposed at different locations on the objects depending on the specific experimental condition. For half of the trials, the circles were located inside the boundaries of the object, and for the other half, one circle was located inside the object and the other was located outside of its boundaries (Figure 4). Critically,

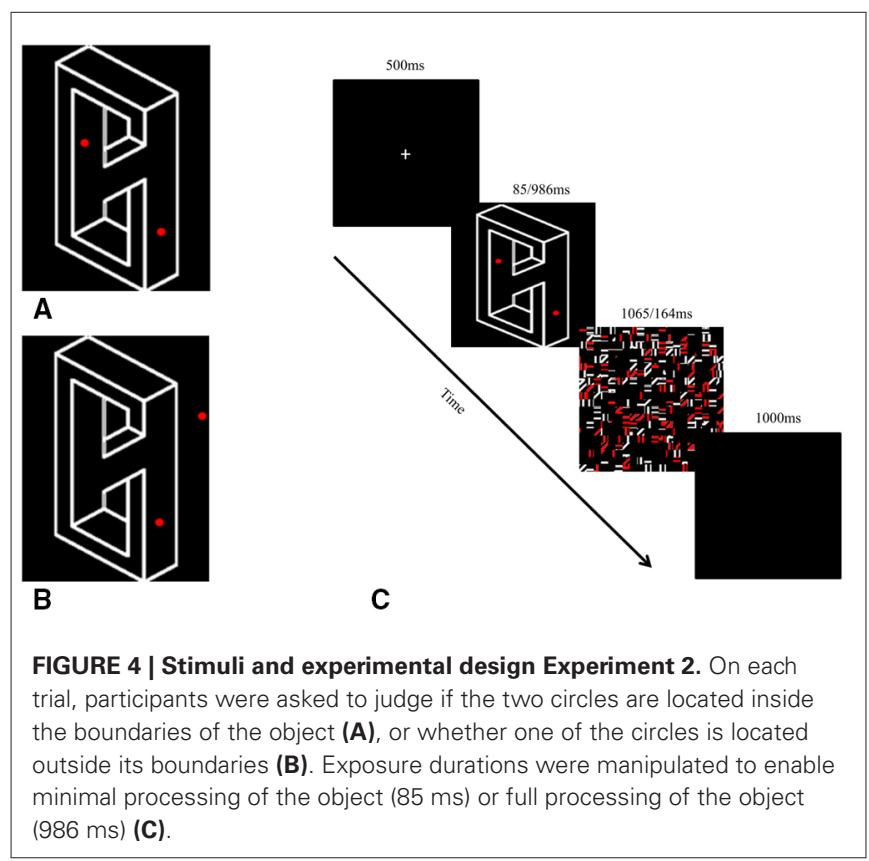

the distance between the two circles was kept constant across trials regardless of their exact position on the screen.

\section{Design}

Object possibility (possible/impossible), exposure duration (85 ms/976 ms), object orientation (aligned /left/right mirror) and correct decision (in/out) served as within-subject variables with each combination occurring in an equal number of trials. The results were collapsed across object orientation and correct decision following a preliminary analyses which ensured that these variables did not interact with possibility or exposure duration $\left[F_{S}<1\right]$. Different exposure durations were presented in separate experimental blocks and the order of blocks was counterbalanced across participants.

An initial analysis of accuracy scores revealed a three-way interaction between exposure duration, possibility, and block order $\left[F_{(1,22)}=3.82, p=0.06, \eta_{p}^{2}=0.14\right]$, implying a carryover effect between blocks. To avoid this effect we discarded the second block of each participant and treated exposure duration as a between-subject variable. Importantly, the overall pattern of the results remains similar between the two analyses.

\section{Experimental procedure}

Participants were asked to judge whether the two circles are located on the object or whether one of the circles is located outside the boundaries of that object (in/out) (Figures 4A,B). The nature of the objects (possible/impossible) was not explicitly mentioned to avoid attentional confounds.

Following four practice trials in which feedback was provided, participants were presented with 448 experimental trials distributed equally and randomly between the different conditions. The trials were self-generated; each trial began with a fixation point $(500 \mathrm{~ms})$, followed by the experimental display (85/986 ms) and a mask that completed the display duration to $1150 \mathrm{~ms}$ (1065, 164 respectively). Trials ended with a blank screen $(1000 \mathrm{~ms}$; Figure 4C). Responses were given on a keyboard, similarly as in Experiment 1.

\section{RESULTS}

The objective of Experiment 2 was to test whether the differences observed in Experiment 1 between possible and impossible objects were mediated by early or by late perceptual processes.

Accuracy and RT were analyzed using repeated measures ANOVA with possibility (possible/impossible) as a within-subject variable and exposure duration $(85 \mathrm{~ms} / 976 \mathrm{~ms})$ as a betweensubject variable. For the RT data, only a main effect of exposure time was observed. RTs were generally faster for the long exposure duration $\left[F_{(1,22)}=4.67, p<0.05, \eta_{p}^{2}=0.17\right]$. No effects were found for possibility or the interaction between exposure duration and possibility [both $F s<1$ ].

The ANOVA for the accuracy data revealed a significant main effect of exposure duration, with more accurate responses in the long exposure duration condition $\left[F_{(1,22)}=13.74, p<0.01\right.$, $\left.\eta_{\mathrm{p}}^{2}=0.38\right]$. More importantly, a significant interaction was found between possibility and exposure durations $\left[F_{(1,22)}=5.43\right.$, $\left.p<0.05, \eta_{p}^{2}=0.2\right]$. Planned comparisons reveled that for the short exposure duration, there were no differences between 


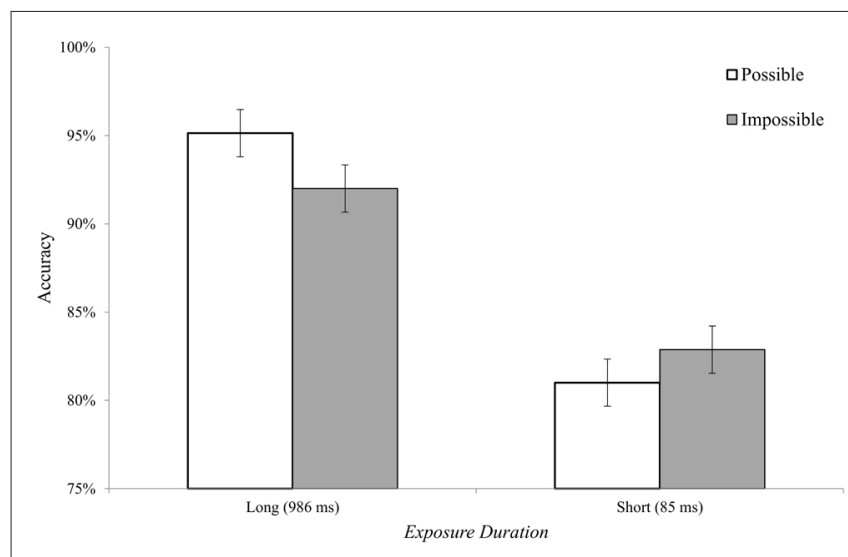

FIGURE 5 | Accuracy data for Experiment 2. Differences between possible and impossible objects were found only for long (986 ms) exposure durations. For short exposure durations (85 ms) no differences were found between the two object types. Error bars represent confidence intervals as calculated for repeated measure ANOVAs for the effect of object possibility (Jarmasz and Hollands, 2009).

possible and impossible objects $\left[F_{(1,22)}=1.88, p>0.15\right]$. On the other hand, for the long exposure duration, performance was more accurate for possible compared to impossible objects, as indicated by the marginally significant planned comparison $\left[F_{(1,22)}=3.69, p=0.06\right.$; Figure 5]. Importantly, the accuracy rate in the short exposure duration was above $80 \%$ and therefore the lack of difference between object categories could not be accounted by a general lack of sensitivity (i.e., floor effect).

Finally, to ensure that in the short exposure duration participants did not apply a strategy of attending one circle while guessing about the location of the second circle, we computed a sensitivity measure ( $d^{\prime}$ score), that took into account response bias separately for possible and impossible objects. Correct responses for the "in" condition (i.e., two circles that were located on the object) were defined as hits, while incorrect responses for the "out" condition (i.e., one circle which was located outside the object) were defined as false alarms. These scores were subjected to a repeated measure ANOVA. A significant interaction was found between possibility and exposure duration $\left[F_{(1,22)}=4.24, p=0.05, \eta_{p}^{2}=0.16\right]$ and planned comparisons showed that the sensitivity was significantly higher for possible objects $\left[d^{\prime}{ }_{\text {possible })}=3.49, d^{\prime}{ }_{\text {impossible })}=3.19\right]$ in the long exposure duration $\left[F_{(1,22)}=4.58, p<0.05\right]$, while no significant differences were observed for the short exposure $\left[F_{(1,22)}<1 ; d^{\prime}\right.$ (possible) $=1.96$, $\left.d^{\prime}{ }_{\text {impossible })}=2.06\right]$. Importantly, the criterion, which reflects a response bias, was not different from 0 regardless of exposure durations $[F$ s $<1]$.

These findings indicate that the perceptual differences between possible and impossible object arise at late perceptual stages while that at initial perceptual stages, the processing of possible and impossible objects is performed in a similar fashion.

\section{GENERAL DISCUSSION}

The results of the current study showed that the representations of possible and impossible objects rely on similar initial perceptual processes, while differentiation between object categories emerge only later along the processing hierarchy.

Experiment 1 demonstrated that impossible objects draw attention automatically and similarly to possible objects, suggesting resemblance in the perceptual processes that mediate the representation of the two object types. Yet, differences in performance were also found between possible and impossible objects, with overall longer reaction times for impossible objects. Previous studies from our lab already showed that possible and impossible objects share similar a initial processing scheme (Freud et al., 2013a,b). In Experiment 1 we validated and extended these findings using a different, well-established paradigm of objectbased attention. Importantly, while previous studies required overt judgments of object dimensions (e.g., size of a particular dimension), here the objects were task-irrelevant and therefore enabled testing whether attention can be allocated in an automatic fashion even for impossible objects.

Experiment 2 was aimed to unravel the source of the overall longer reaction times that were found for impossible objects in Experiment 1. The results indicate that the differences between possible and impossible object emerge only when objects were presented for long exposure duration, and therefore are probably affected by late perceptual processes, such as the rendering of fine-detailed description of object structure (Hochstein and Ahissar, 2002). Importantly, the level of processing is inevitable, and participants could not "choose" to process only the gist of the object. Presumably, in the long exposure duration the visual system automatically attempted to generate a fine-detailed description of the impossible objects, however the incoherency of $3 \mathrm{D}$ structure could have impaired this process, and therefore the task (which relied on the processing of the object) was performed less accurately compared to situations in which the fine-grained representation could be successfully generated. These results converge with a recent study from our lab that used the Garner's speeded classification task to unravel the perceptual representation of possible and impossible objects. In this study the level of processing was manipulated by filtering-out specific spatial frequency ranges and not by limiting exposure duration as in Experiment 2. Interestingly, it was found that when low spatial frequency (LSF) information, which usually supports rapid, coarse representation (e.g., Bar et al., 2006), was available, similar processing style was observed for possible and impossible objects. On the other hand, when LSF information was filtered out, only HSF information was available which could support late, but detailed processing of object shape. Under this condition, a dissociation was found between the object categories. Specifically, possible objects were still processed in a holistic unified manner, while impossible objects were no longer processed as unified objects (Freud et al., 2014).

The investigation of the representation of impossible objects could shed light on the cognitive and neural mechanisms that mediate the perception of novel (possible) objects in everyday life. In particular, the results of the present study could be taken as a general support for the reverse hierarchy theory (RHT; Hochstein and Ahissar, 2002) in the context of $3 \mathrm{D}$ information representation. In a nutshell, the RHT theory 
proposes that visual perception is based on two main stages. First, a feed-forward processing stream represents the "gist of the scene" while the exact details are not fully encoded. The second stage is based on recurrent processing that relies on low-level regions in the visual cortex and enables the encoding of detailed information.

According to the RHT, object-based attention is accomplished by fast feed forward processing. Thus, the similar gist, which characterized matched possible and impossible objects, could have mediated the equivalent object-based attention results obtained in Experiment 1. Similarly, in Experiment 2, when objects were presented for a short duration, the task could have been performed based on the gist of the objects while the fine details were not accessible. This could account for the similar performance patterns obtained for the two object categories under this condition. On the other hand, when objects were presented for longer durations, feedback processing could have taken place and the fine details that define spatial ambiguity of impossible objects could be processed, which in turn led to reduced accuracy for impossible objects.

The perceptual processes that mediate the representation of impossible objects could also be discussed in light of the Bayesian framework. According to this framework, information conveyed by the stimulus is represented by the visual system as a conditional probability density function. The visual system uses past experience to compute the most probable interpretation given the retinal image stimulus (Kersten et al., 2004; Knill and Pouget, 2004). This conceptualization was developed by behavioral and computational studies that demonstrated that Bayesian models nicely fit to the perceived experience in a variety of perceptual phenomena, including depth perception which follows the integration of multiple depth cues (e.g., van Ee et al., 2003) and visual illusions of motion (Geisler and Kersten, 2002; Weiss et al., 2002). Although the present investigation was not aimed to test whether a Bayesian framework could account for the representation of impossible objects, it is plausible that the visual system erroneously estimates the likelihood of different depth cues (global/local) which could lead to an incoherent percept of the stimulus. Such a process may also mediate the behavioral advantage observed for possible objects in Experiment 1 and in the long exposure duration of Experiment 2. Future studies could use this framework to further unravel how the brain estimates and weights different types of monocular depth cues in a Bayesian fashion.

To summarize, our findings suggest that initially, possible and impossible objects are spatially organized in a similar manner and that impossible figures are represented in a similar manner to possible ones at least in terms of capturing automatic visual attention. The visual system successfully utilizes intact shape attributes to create a successful representation of impossible objects. These properties override the odd spatial layout inherent to impossible objects while the phenomenological differences between object-types may reflect high-level perceptual processes that occur later in time. Yet, the nature of these processes needs to be elaborated by future research that will examine their dynamics and the cognitive and the neural correlates mediating these processes.

\section{REFERENCES}

Bar, M. (2003). A cortical mechanism for triggering top-down facilitation in visual object recognition. J. Cogn. Neurosci. 15, 600-609. doi: 10.1162/ 089892903321662976

Bar, M., Kassam, K. S., Ghuman, A. S., Boshyan, J., Schmid, A. M., Dale, A. M., et al. (2006). Top-down facilitation of visual recognition. Proc. Nat. Acad. Sci. U.S.A. 103, 449-454. doi: 10.1073/pnas.0507062103

Burge, J., Peterson, M. A., and Palmer, S. E. (2005). Ordinal configural cues combine with metric disparity in depth perception. J. Vis. 5, 5. doi: 10.1167/5.6.5

Di Luca, M., Domini, F., and Caudek, C. (2004). Spatial integration in structure from motion. Vision Res. 44, 3001-3013. doi: 10.1016/j.visres.2004.07.004

Domini, F., Caudek, C., and Richman, S. (1998). Distortions of depth-order relations and parallelism in structure from motion. Percept. Psychophys. 60, 1164-1174. doi: 10.3758/BF03206166

Domini, F., Caudek, C., and Tassinari, H. (2006). Stereo and motion information are not independently processed by the visual system. Vision Res. 46, 1707-1723. doi: 10.1016/j.visres.2005.11.018

Drummond, L., and Shomstein, S. (2010). Object-based attention: shifting or uncertainty? Atten. Percept. Psychophys. 72, 1743-1755. doi: 10.3758/APP.72.7. 1743

Duncan, J. (1984). Selective attention and the organization of visual information. J. Exp. Psychol. Gen. 113, 501-517. doi: 10.1037/0096-3445.113.4.501

Freud, E., Avidan, G., and Ganel, T. (2013a). Holistic processing of impossible objects: evidence from Garner's speeded-classification task. Vision Res. 93, 1018. doi: 10.1016/j.visres.2013.10.001

Freud, E., Ganel, T., and Avidan, G. (2013b). Representation of possible and impossible objects in the human visual cortex: evidence from fMRI adaptation. Neuroimage 64, 685-692. doi: 10.1016/j.neuroimage.2012.08.070

Freud, E., Avidan, G., and Ganel, T. (2014). The highs and lows of object impossibility: effects of spatial frequency on holistic processing of impossible objects. Psychon. Bull. Rev. doi: 10.3758/s13423-014-0678-2 [Epub ahead of print].

Freud, E., Rosenthal, G., Ganel, T., and Avidan, G. (2015). Sensitivity to object impossibility in the human visual cortex: evidence from functional connectivity. J. Cogn. Neurosci. doi: 10.1162/jocn_a_00753 [Epub ahead of print].

Garner, W. and Felfoldy, G. L. (1970). Integrality of stimulus dimensions in various types of information processing. Cogn. Psychol. 1, 225-241. doi: 10.1016/00100285(70)90016-2

Geisler, W., Perry, J., Super, B., and Gallogly, D. (2001). Edge co-occurrence in natural images predicts contour grouping performance. Vision Res. 41, 711-724. doi: 10.1016/S0042-6989(00)00277-7

Geisler, W. S., and Kersten, D. (2002). Illusions, perception and Bayes. Nat. Neurosci. 5, 508-510. doi: 10.1038/nn0602-508

Goldfarb, L., and Tzelgov, J. (2005). Is size perception based on monocular distance cues computed automatically? Psychon. Bull. Rev. 12, 751-754. doi: 10.3758/ BF03196768

Grill-Spector, K., and Kanwisher, N. (2005). Visual recognition: as soon as you know it is there, you know what it is. Psychol. Sci. 16, 152-160. doi: 10.1111/ j.0956-7976.2005.00796.x

Habeck, C., Hilton, H. J., Zarahn, E., Brown, T., and Stern, Y. (2006). An eventrelated fMRI study of the neural networks underlying repetition suppression and reaction time priming in implicit visual memory. Brain Res. 1075, 133-141. doi: 10.1016/j.brainres.2005.11.102

Hochstein, S., and Ahissar, M. (2002). View from the top: hierarchies and reverse hierarchies in the visual system. Neuron 36, 791-804. doi: 10.1016/S08966273(02)01091-7

Jarmasz, J., and Hollands, J. G. (2009). Confidence intervals in repeated-measures designs: the number of observations principle. Can. J. Exp. Psychol. 63, 124-138. doi: $10.1037 / \mathrm{a} 0014164$

Jiang, X., Rosen, E., Zeffiro, T., VanMeter, J., Blanz, V., and Riesenhuber, M. (2006). Evaluation of a shape-based model of human face discrimination using fMRI and behavioral techniques. Neuron 50, 159-172. doi: 10.1016/j.neuron. 2006.03.012

Kersten, D., Mamassian, P., and Yuille, A. (2004). Object perception as Bayesian inference. Annu. Rev. Psychol. 55, 271-304. doi: 10.1146/annurev.psych.55. 090902.142005

Kimchi, R., Yeshurun, Y., and Cohen-Savransky, A. (2007). Automatic, stimulusdriven attentional capture by objecthood. Psychon. Bull. Rev. 14, 166-172. doi: 10.3758/BF03194045 
Knill, D. C. (2007). Learning Bayesian priors for depth perception. J. Vis. 7, 13. doi: $10.1167 / 7.8 .13$

Knill, D. C., and Pouget, A. (2004). The Bayesian brain: the role of uncertainty in neural coding and computation. Trends Neurosci. 27, 712-719. doi: 10.1016/j.tins.2004.10.007

Koffka, K. (1935). Principles of Gestalt Psychology. New York, NY: Harcourt, Brace and World.

Kovacs, I., and Julesz, B. (1993). A closed curve is much more than an incomplete one: effect of closure in figure-ground segmentation. Proc. Natl. Acad. Sci. U.S.A. 90, 7495-7497. doi: 10.1073/pnas.90.16.7495

Marr, D. (1982). Vision: A Computational Investigation into the Human Representation and Processing of Visual Information. New York, NY: Henry Holt and Co., Inc.

Moore, C. M., Yantis, S., and Vaughan, B. (1998). Object-based visual selection: evidence from perceptual completion. Psychol. Sci. 9, 104-110. doi: 10.1111/14679280.00019

Naber, M., Carlson, T. A., Verstraten, F. A. J., and Einhäuser, W. (2011). Perceptual benefits of objecthood. J. Vis. 11, 8. doi: 10.1167/11.4.8

Penrose, L. S., and Penrose, R. (1958). Impossible objects: a special type of visual illusion. Br. J. Psychol. 49, 31-33. doi: 10.1111/j.2044-8295.1958.tb00634.x

Regolin, L., Rugani, R., Stancher, G., and Vallortigara, G. (2011). Spontaneous discrimination of possible and impossible objects by newly hatched chicks. Biol. Lett. 7, 654-657. doi: 10.1098/rsbl.2011.0051

Sayres, R., and Grill-Spector, K. (2006). Object-selective cortex exhibits performance-independent repetition suppression. J. Neurophysiol. 95, 9951007. doi: $10.1152 /$ jn. 00500.2005

Shuwairi, S. M., Albert, M. K., and Johnson, S. P. (2007). Discrimination of possible and impossible objects in infancy. Psychol. Sci. 18, 303-307. doi: 10.1111/j.14679280.2007.01893.x
Sugase, Y., Yamane, S., Ueno, S., and Kawano, K. (1999). Global and fine information coded by single neurons in the temporal visual cortex. Nature 400, 869-873.

van Ee, R., Adams, W. J., and Mamassian, P. (2003). Bayesian modeling of cue interaction: bistability in stereoscopic slant perception. J. Opt. Soc. Am. A Opt. Image Sci. Vis. 20, 1398-1406. doi: 10.1364/JOSAA.20.001398

Weiss, Y., Simoncelli, E. P., and Adelson, E. H. (2002). Motion illusions as optimal percepts. Nat. Neurosci. 5, 598-604. doi: 10.1038/nn0602-858

Zemel, R. S., Behrmann, M., Mozer, M. C., and Bavelier, D. (2002). Experiencedependent perceptual grouping and object-based attention. J. Exp. Psychol. Hum. Percept. Perform. 28, 202-217. doi: 10.1037/0096-1523.28.1.202

Conflict of Interest Statement: The authors declare that the research was conducted in the absence of any commercial or financial relationships that could be construed as a potential conflict of interest.

Received: 10 October 2014; accepted: 17 January 2015; published online: 16 February 2015.

Citation: Freud E, Hadad B-S, Avidan G and Ganel T (2015) Evidence for similar early but not late representation of possible and impossible objects. Front. Psychol. 6:94. doi: 10.3389/fpsyg.2015.00094

This article was submitted to Perception Science, a section of the journal Frontiers in Psychology.

Copyright $\odot 2015$ Freud, Hadad, Avidan and Ganel. This is an open-access article distributed under the terms of the Creative Commons Attribution License (CC BY). The use, distribution or reproduction in other forums is permitted, provided the original author(s) or licensor are credited and that the original publication in this journal is cited, in accordance with accepted academic practice. No use, distribution or reproduction is permitted which does not comply with these terms. 